\title{
Hausärztliche Highlights vom europäischen Kardiologenkongress 2007
}

HOHE MORTALITÄT BEI PAVK

\section{Auch asymptomatische Patienten sterben früher}

- Durch eine periphere arterielle Verschlusskrankheit (PAVK) wird die Lebenszeit stark verkürzt, auch dann wenn die Erkrankung keine Beschwerden verursacht. In der getABI-Studie, einer epidemiologischen Untersuchung aus Deutschland, waren fünf Jahre nach der Diagnose einer PAVK 23,9\% der Patienten mit Symptomen und $19,1 \%$ der beschwerdefreien Patienten gestorben, im Vergleich zu 9,4\% der Patienten ohne PAVK. Nach Aussage des Studienleiters Prof. Curt Diehm aus Heidelberg ist der Unterschied zwischen symptomatischen und asymptomatischen PAVK-Patienten nicht signifikant. Entscheidend für die Lebenserwartung sei vielmehr der mit dem KnöchelArm-Index (ABI) gemessene Schweregrad der peripheren Atherosklerose.
Diehm rief deswegen dazu auf, bei älteren Patienten regelmäßig den $A B I z u$ bestimmen und bei Diagnose einer PAVK sekundärpräventive Maßnahmen einzuleiten.

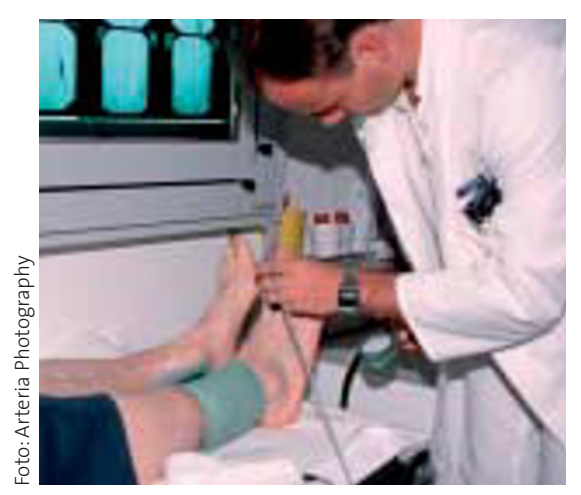

Je niedriger der $A B I$, desto höher die Sterblichkeit.

\section{SEKUNDÄRPRÄVENTION BEI KHK}

\section{Die Therapie wird besser, der Lebensstil ungesünder}

-Wie gut ist die Behandlung von KHKPatienten in Europa? Dieser Frage sind die Studien EUROASPIRE I bis III von den frühen 199oer-Jahren bis zu den Jahren 2006/2007 nachgegangen. Für neun Länder, darunter auch Deutschland, wurde jetzt die Entwicklung über die letzten 15 Jahre angeschaut. Die gute Nachricht: Die Ärzte machen ihre Arbeit immer besser, die allermeisten Patienten erhalten heute die empfohlene medikamentöse Sekundärprävention, berichtete Studienautor Prof. David A. Wood aus London.
Die schlechte Nachricht: Die Patienten halten immer seltener einen gesunden Lebensstil ein. So ist der Anteil von adipösen Koronarkranken von früher $25 \%$ auf inzwischen $38 \%$ angestiegen. Das schlägt sich nieder in einer Zunahme der Diabetes-mellitus-Erkrankungen von $17 \%$ auf $28 \%$. Die Raucherquote unter den KHK-Patienten liegt unverändert bei etwa $20 \%$. Diese Befunde erklären wahrscheinlich auch, warum zum Beispiel die Blutdruckwerte der Patienten trotz besserer Therapie unverändert schlecht sind.

\section{NACH DEM RAUCHVERBOT IN IRLAND}

\section{Schlagartiger Rückgang der Herzinfarkte}

- Während man in Deutschland noch immer über die genauen Bedingungen für Rauchverbote diskutiert, können andere Länder längst beeindruckende Erfolge dieser Maßnahme vorweisen. Zum Beispiel Irland: Als erstes EU-Land hat es im Jahr 2004 ein generelles Rauchverbot am Arbeitsplatz und an allen öffentlichen Orten eingeführt. Bereits im ers- ten Jahr danach ging die Zahl der Krankenhauseinweisungen wegen akuter Koronarsyndrome um rund $11 \%$ zurück. Dass sich die saubere Luft bereits nach so kurzer Zeit bemerkbar macht, kommt für die Autoren der Registerstudie nicht überraschend. Auch nach einer Raucherentwöhnung werde das Herzinfarktrisiko schnell kleiner.

\section{HERZINSUFFIZIENZ-PARADOXON}

\section{Dicke leben länger}

Wenn Ihre Herzinsuffizienzpatienten übergewichtig sind, sollten Sie sie nicht zum Abspecken animieren, solange ihr BMI noch unter $40 \mathrm{~kg} / \mathrm{m}^{2}$ liegt. Prof. Stefan Anker aus Berlin begründete diese überraschende Aussage mit Daten von mehr als 30000 Herzinsuffizienzpatienten. Sie zeigen eindeutig, dass dicke Patienten die beste Prognose hinsichtlich Morbidität und Überlebenszeit haben. Gewichtsverlust ist dagegen ein schlechtes prognostisches Zeichen. Die Datenlage rechtfertige es aber noch nicht, herzinsuffizienten Patienten eine Gewichtszunahme anzuraten, so Anker.

\section{MEDIKAMENTENBESCHICHTETE STENTS}

\section{Wie sicher sind sie?}

Medikamentenbeschichtete Stents (Drug Eluting Stents, DES) haben den Vorteil, wirksamer vor Restenosen zu schützen als nackte Metallstents. Seit einiger Zeit gibt es jedoch Debatten über ihre Sicherheit, da in seltenen Fällen lebensgefährliche späte Stentthrombosen auftreten. Wie das langfristige Nutzen-Risiko-Profil aussieht, war jetzt Gegenstand zweier Registerstudien. In der größeren Studie wurden fast 120000 Stentpatienten erfasst, davon fast ein Drittel mit DES. Über die Beobachtungszeit von vier Jahren gab es hier keinen Unterschied zwischen den beiden Gruppen in Bezug auf die Herzinfarkt- oder Sterberate. „Das sind gute Neuigkeiten für die Patienten. Es gibt zwar ein erhöhtes Risiko für späte Stentthrombosen, das sich aber nicht in einer höheren Infarktrate oder Mortalität niederschlägt ", sagte Studienautor Prof. Stefan James aus Uppsala.

Bei Patienten mit akutem ST-Hebungsinfarkt sollten jedoch besser keine DES zum Einsatz kommen, so die Einschätzung von Prof. Philipp G. Steg aus Paris. In einer von ihm präsentierten 2-JahresStudie hatten diese Patienten ab dem sechsten Monat eine sechsfach höhere Sterblichkeit als Patienten mit herkömmlichen Stents.

BS - 